[Contribution from the Fixed Nitrogen Research Laboratory, Wak DepartMENT AND THE BUREAU OF SOILS, DEPARTMENT OF AGRICULTURE.]

\title{
THE PHOTOCHEMICAL DECOMPOSITION OF NITROGEN PENTOXIDE.
}

By Farrington Danieis and Eimer H. Johnston.

Received October 9, 1920.

It was shown in a previous communication ${ }^{1}$ that the decomposition of nitrogen pentoxide is a monomolecular, gas-phase reaction which takes place with convenient velocity at room temperature. Such a system is peculiarly valuable in studying the relation between thermal and photochemical action, and in testing the new theories ${ }^{2}$ which connect activity with the absorption of radiant energy.

It was shown in this preceding research that the "critical increment" for the decomposition of nitrogen pentoxide has very closely the value 24,700 calories or $1.031 \times 10^{12}$ ergs over the temperature range $0^{\circ}$ to $65^{\circ}$. In accordance with the theories of Lewis and Perrin "critical increment" $E$ and the frequency $\nu$ of the radiant energy which brings about the reaction should be connected by the equation,

$$
E=N h \nu
$$

where $N$ is the number of molecules in a mol and $h$ is Planck's constant. The significance of $\nu$, the frequency of the activating radiant energy is a matter of great interest. According to W. C. M. Lewis and to Perrin, ${ }^{3}$ it is practically a single frequency, the head of an absorption band.

Inserting the value of $E$ obtained in the previous research we have,

$$
\nu=\frac{E}{N h}=\frac{1.031 \times 10^{12}}{6.1 \times 10^{23} \times 6.56 \times 10^{-27}}=2.58 \times 10^{14} .
$$

Changing frequencies, $\nu$, into wave lengths, $\mu$, by dividing into the velocity of light, we obtain,

$$
\frac{3.00 \times 10^{10}}{2.58 \times 10^{14}}=1.16 \times 10^{-4} \mathrm{~cm} .=1.16 \mu .
$$

It follows from these theories that if energy of this frequency, of greater density than that emitted by thermal radiation from the walls of the containing vessel acts on nitrogen pentoxide the decomposition rate should be increased. Fortunately there is a considerable amount of energy of this frequency in sunlight or in light from an electric lamp, since it lies in the infra-red region, near the visible. Moreover, glass and water, in thin

1 Preceding article, This Journal, 43, 53 (1921).

2 Trautz, Z. anorg. Chem, I02, 81 (1918) and preceding pages; Haber, Verh. deut. physik. Ges., I3, 1117 (1911); W. C. M. Lewis, J. Chem. Soc., Ir3, 471 (1918) and preceding pages; Phil. Mag., 39, 26 (1920); Perrin, "Les Atoms" (1913); Ann. phys., II, 5 (1919).

Loc, cit. 
layers are nearly transparent to light of wave length $1.16 \mu$. It is, of course, necessary to exclude from consideration, decomposition caused by the thermal radiation of the vessel walls in order to determine the effect of radiant energy from an external source. Fortunately, again this condition can be easily realized in the laboratory. From the previous research it is seen that at $0^{\circ}$, or below, the thermal decomposition is negligible over a period of an hour or so. By merely packing a glass vessel containing ritrogen pentoxide in ice and water, we have a system in which photochemical action can be distinguished from thermal action.

It should be emphasized that there is no fundamental difference in the mechanism of photochemical and thermal reactions, but in a photochemical reaction the radiating body is not in thermal equilibrium with the reacting substance, as it is in a thermal reaction, and the distribution of energy among the different frequencies does not necessarily follow Planck's distribution law. The term, photochemical reaction, has sometimes been taken to mean a reaction which is brought about by visible or near-visible light, but it would be better to define a photochemical reaction merely as one which is brought about by radiant energy from an external source which is not in equilibrium with the reacting system.

The photochemical cell used in the present research was a small glass vessel, with a bulb of thin blown glass for the admission of light and 2 side arms by means of which nitrogen pentoxide was introduced. The preparation and purification of this material have been described, and, as before, the crystals were subjected to continued evacuation before sealing off. Since the solid phase was present, the pressure of gaseous nitrogen pentoxide was always equal to $51 \mathrm{~mm}$., the vapor pressure at $0^{\circ}$. A glass manometer of the type used in the previous researches was fused to the cell. With the exception of the window of thin blown glass, the whole apparatus was painted black.

The cell was packed in ice and water in a pail, and set so that the window was turned toward the sun and close to the surface of the ice-bath. The intensity of the sunlight was obtained, through the kindness of Mr. G. H. Hand, from the continuous records of the Solar Radiation Laboratory of the Weather Bureau, situated a short distance away. In about half of the experiments, light from a 500 -watt tungsten lamp set at a distance of about $15 \mathrm{~cm}$. was used in place of sunlight. Variations in the intensity of light, its angle of incidence, and the ratio of exposed surface to volume in the different cells prevented accurate quantitative measurements.

The pressure increase in bright sun usually amounted to about $20 \mathrm{~mm}$. per hour, with a window of $4 \mathrm{sq} . \mathrm{cm}$. and a volume of approximately $25.0 \mathrm{cc}$. The pressure readings were accurate to a millimeter or less. As a rule the gas alone was subjected to the action of the light, but the presence of solid crystals of nitrogen pentoxide in its path made no difference. 
Several different cells were used and about 75 experiments were carried out. The general conclusions are given in the following paragraphs.

Within the Limits of Experimental Accuracy, Nitrogen Pentoxide is not Decomposed by Light in the Region of $I . I 6 \mu$.-In 2 experiments the photochemical cell in an ice-bath, was exposed to bright sunlight for an hour through a screen of $2 \mathrm{~cm}$. of a saturated solution of iodine in carbondisulfide, without any detectable decomposition. Such a screen is opaque to visible light, but transparent to the infra-red including the region in question. ${ }^{1}$ In another experiment a copper tube filled with the carbon disulfide solution of iodine was cemented over the window and exposed to a 500-watt tungsten lamp at a distance of $15 \mathrm{~cm}$. between the hot filament and the window. The only materials in the path of the light were 2 very thin pieces of glass and the carbon disulfide solution of iodine, but the pressure in the cell did not rise during an hour's exposure, by as much as a millimeter, which was the limit of accuracy of the manometer. As an additional check on the fact that light of the predicted wave length does not increase the rate of decomposition, the results of several experiments without the iodine screen showed that although the decomposition of nitrogen pentoxide is accelerated by unobstructed light, the rate is the same within the limits of experimental error, whether the whole of the sun rays, including wave length $1.16 \mu$, which lies in the infra-red, or only the visible portion is admitted. In one experiment no screen was used and the window was allowed to protrude from the ice-bath, in a second the light was passed through $5 \mathrm{~cm}$. of water; and in a third, a screen of $0.4 \mathrm{~N}$ cupric chloride solution was interposed. The rates of decomposition were the same, although in the first case there was no absorption of the sun's energy in the visible or infra-red, in the second only $20 \%$ of the light of wave length $1.16 \mu$ was transmitted, and in the third case the screen was practically opaque to light of longer wave length than $600 \mu \mu$.

It should be distinctly emphasized that this result is in direct contradiction to the theories of W. C. M. Lewis and Perrin. It does not disagree, however, with the theoretical work of Tolman.2

Blue Light Accelerates the Decomposition of Nitrogen Pentoxide.-Visible light was found to have a pronounced effect on the decomposition of nitrogen pentoxide. This was to be expected from the observations of Berthelot ${ }^{3}$ and from the fact that concentrated nitric acid is so readily decomposed by sunlight. The frequency of the light which was responsible for the reaction was determined by means of screens of copper chloride -calcium chloride solutions, which give sharply defined transparent regions from $600 \mu \mu$ to $500 \mu \mu$ and shorter, depending on the proportion

1 Coblentz, Phys. Rev., 16, 35, 72 (1903).

2 Tolman, ThIs Journat, 42, 2506 (1920).

${ }^{3}$ Ann. Chem. Phys, , 6, 202 (1875). 
of calcium chloride. Experiments showed that a screen $2 \mathrm{~cm}$. thick of solution of $0.4 \mathrm{~N}$ cupric chloride, $2.55 \mathrm{~N}$ calcium chloride stopped the photochemical action completely. According to measurements of Uehler and Jones ${ }^{1}$ this filter transmits light only between $600 \mu \mu$ and $460 \mu \mu$. Screens with a higher portion of calcium chloride and a correspondingly smaller region of transparency were just as effective in stopping the photochemical decomposition. A screen of $0.4 \mathrm{~N}$ cupric chloride and $0.85 \mathrm{~N}$ calcium chloride solution, however, had no effect in retarding the photochemical action. This filter is transparent between $600 \mu \mu$ and $400 \mu \mu$. It is evident then that only the light between $400 \mu \mu$ and $460 \mu \mu$ (blue light) is effective in accelerating the decomposition of nitrogen pentoxide. No conclusions regarding the action of light shorter than $400 \mu \mu$ (ultraviolet) can be drawn because the glass itself becomes opaque in this region and because there is very little of this light in sunlight or in light from a tungsten filament.

Three screens in which the calcium chloride concentrations were such that the lower limit of transparency varied between $400 \mu \mu$ and $460 \mu \mu$ permitted photochemical decomposition but to a lessened degree. A screen containing a saturated solution of $p$-nitroso-dimethyl aniline permitted but slight action, the pressure rising about $3 \mathrm{~mm}$. an hour in bright sunlight. This screen is opaque to light in the general region from $380 \mu \mu$ to $450 \mu \mu$, but the boundaries are not sharp. ${ }^{2}$

Bine Light Does not Decompose Nitrogen Pentoxide, unless Nitrogen Dioxide is Present.--In other words, the brown nitrogen dioxide acts as an auto-catalyst in blue light. The fact that there is practically complete absorption by nitrogen dioxide ${ }^{3}$ of the light which is effective in accelerating the decomposition of nitrogen pentoxide led to the suspicion that the dioxide (one of the decomposition products) was taking a part in the action. Accordingly a screen of nitrogen dioxide gas was placed in the path of the light, with the result that the photochemical decomposition was completely stopped. No precautions had been taken in sealing off the capillaries and in all cases there was some dioxide present, the amount of which could be calculated by subtracting the vapor pressure of pentoxide from the total pressure, and allowing for dissociation of nitrogen tetroxide by the methods of the previous article. The following table shows how the photochemical decomposition depends on the quantity of dioxide present. No screens were used in the experiments recorded here. The area of the window was about $4 \mathrm{sq}$. cm. so that multiplying the sun's radiation by 4 gives the energy admitted.

${ }^{\prime}$ Uehler and Jones, Carnegie Inst. Pub., 6o, 213.

2 Vehler and Wood, ibid., 7I, 21.

${ }^{3}$ Hasselberg, Mem, acad. St. Petersburg, (7) 26, 286 (1878). 


\begin{tabular}{|c|c|c|c|c|c|}
\hline \multicolumn{2}{|r|}{ Time. } & $\begin{array}{c}\text { Sun's } \\
\text { radiation. } \\
\text { Calories } \\
\text { per hour } \\
\text { per sq. cm. }\end{array}$ & $\begin{array}{c}\text { Pressure } \\
\mathrm{NO}_{2} \\
\text { at start. } \\
\mathrm{Mm} .\end{array}$ & $\begin{array}{c}\text { Pressure } \\
\mathrm{N}_{2} \mathrm{O}_{3} \\
\text { decomposed } \\
\text { per hour. } \\
\mathrm{Mm} .\end{array}$ & $\begin{array}{c}\text { Pressure } \\
\mathrm{N}_{2} \mathrm{O} \\
\text { decomposed } \\
\text { per hour } \\
\text { per calorie. } \\
\text { Mm. }\end{array}$ \\
\hline \multirow[t]{5}{*}{ June } & $1812.17-1.17$ & 66 & 5 & 2 & 0.01 \\
\hline & $18 \quad 2.18-3.18$ & 55 & 9 & 4 & 0.02 \\
\hline & $18 \quad 3.48-4.48$ & 34 & 13 & 4 & 0.03 \\
\hline & $2410.06-11.06$ & 76 & 26 & 10 & 0.03 \\
\hline & $25 \quad 3.35-4.20$ & 54 & 38 & $1 \tilde{5}$ & 0.07 \\
\hline \multirow[t]{2}{*}{ July } & $1 \quad 1.30-2.30$ & 72 & 56 & 17 & 0.06 \\
\hline & $311.00-11.35$ & 77 & 64 & 24 & 0.08 \\
\hline
\end{tabular}

A comparison of the third and fifth columns shows that when the amount of nitrogen dioxide is small the photochemical decomposition is slight, and as the amount increases the action goes faster. The effect of further dioxide falls off, however, as the concentration increases

$\begin{array}{cc}\mathrm{NO}_{2} \text { present. } & \text { TABLE II. } \\ \text { Pressure No. } \\ \text { Mm. } & \text { Corrected pressure increase in } 10 \text { mins. } \\ \text { Mm. } & \mathrm{N}_{2} \mathrm{O}_{3} \text { decomposed. } \\ 0 & 0 \\ 13 & 2.7 \\ 32 & 5.0 \\ 1 & 0.0 \\ 10 & 1.3 \\ 13 & 3.9 \\ 0.4 & 0.3 \\ 14 & 1.6 \\ 16 & 3.0 \\ 18 & 3.9 \\ 21 & 2.8 \\ 23 & 6.2 \\ 26 & 7.0 \\ 28 & 8.5 \\ 29 & 5.3 \\ 8 & 1.2 \\ 17 & 3.5 \\ 19 & 5.1 \\ 3 & 1.1 \\ 0.7 & 0.5 \\ 5 & 1.5 \\ 22 & 3.5 \\ 25 & 3.1 \\ 1.4 & 0.2 \\ 2.8 & 0.5\end{array}$

In order to check this result further and to carry out experiments in which every trace of nitrogen dioxide could be eliminated, another type of cell was used, in which a stopcock took the place of the sealed-off capillaries. The action of nitrogen pentoxide on stopcock grease at $0^{\circ}$ over short periods of time was negligible. The crystals were closed off by the 
stopcock after continued evacuation, while the whole apparatus was immersed in an ice-bath. Thermal decomposition gave only about $0.1 \mathrm{~mm}$. of nitrogen dioxide per hour at $0^{\circ}$. In these experiments the cell consisted of a large tube set horizontally one $\mathrm{cm}$. below the surface of the ice-bath, exposing an area of nearly $30 \mathrm{sq}$. cm. A 500-watt tungsten lamp was set at a distance of about $10 \mathrm{~cm}$. The quantity of nitrogen dioxide was varied by warming the cell or by evacuating it. The results are given in lable II, arranged in chronological order, each exposure being ten minutes.

The results show, within the limits of experimental error, that when all of the nitrogen dioxide is removed, light in the general region between 400 and $2000 \mu \mu$ does not decompose nitrogen pentoxide. As noted before, increasing amounts of dioxide give greater decomposition, but the effect becomes less at the higher concentration. The results are qualitative rather than quantitative, since no special attempt was made to keep the voltage of the lamp nor its position constant.

Nitrogen Dioxide Is not an Auto-catalyst in the Dark.-The results of the previous research show that the thermal decomposition is strictly monomolecular, whereas if nitrogen dioxide acted as a catalyst the reaction velocity constant would increase, as the quantity of dioxide increased. Furthermore, when every trace of dioxide was removed and exposure to light caused no decomposition, still the decomposition rate as determined over the succeeding 24 hours at $0^{\circ}$ in a dark box was identical with that which had been obtained in the previous research, and which was always obtained regardless of the quantity of dioxide present.

To prove that the photochemical decomposition was not due to elevation of the temperature by absorption of the light, an experiment was carried out in which careful pressure observations were made while light entered the cell intermittently. No change in pressure, whatever, could be detected when brilliant sunlight was suddenly admitted to the chamber. A change of $0.5 \mathrm{~mm}$. could have been easily detected in this way, corresponding to a rise in temperature of $3^{\circ}$ throughout the gas. To account for the increase in pressure on the basis of thermal decomposition (black body radiation) would require, according to the data of the previous communication, that a temperature of about $15^{\circ}$ above that of the icebath was maintained continuously.

One experiment to determine roughly the amount of energy absorbed in the photochemical decomposition was carried out with the help of Mr. G. H. Hand, of the Solar Radiation Laboratory of the Weather Bureau. On an unusually clear day it was found that the $0.4 N$ cupric chloride$0.85 N$ calcium chloride solution which had a transparent region between 400 and $600 \mu \mu$ let through $22 \%$ of the sun's energy, and had no retarding effect on the photochemical decomposition. A second screen of $0.4 \mathrm{~N}$ 
cupric chloride-2.55 $N$ calcium chloride, transmitting only between 460 and $600 \mu \mu$, allowed but $16 \%$ of the total radiation to pass through, and stopped the decomposition completely. Only $6 \%$ of the sun's energy, then, was effective in bringing about the reaction. It is to be pointed out that this $6 \%$ is a maximum value and that with more experiments the limits might have been pushed closer together. With the first screen the pressure rose $18 \mathrm{~mm}$. in an hour, during which time the radiation rereceived from the sun amounted to 74 calories. Since the area of the exposed window was about $4 \mathrm{sq}$. cm. the total energy entering the cell was 296 calories, and of this less than 18 calories $(6 \%$ ) were capable of bringing about any reaction.

It is interesting to compare this value with the theoretical absorption calculated from the critical increment. In accordance with the theories of Lewis and Perrin, each molecule in order to decompose must absorb the critical increment divided by Avogadro's number,

$$
\frac{E}{N}=\frac{2.47 \times 10^{4}}{6.1 \times 10^{23}}=4.05 \times 10^{-20} \mathrm{cals} \text {. }
$$

In the experiment already described the observed pressure increased from $186.4 \mathrm{~mm}$. to $204.2 \mathrm{~mm}$. during the exposure. Since the volume was $25 \mathrm{cc}$. and the pressure change, corrected for the $\mathrm{N}_{2} \mathrm{O}_{4} \longleftrightarrow 2 \mathrm{NO}_{2}$ equilibrium, $10 \mathrm{~mm}$., it can be calculated that the number of molecules of pentoxide decomposed was $1.0 \times 10^{19}$. Multiplying the energy absorbed per molecule by $1.0 \times 10^{19}$ gives 0.4 calorie, the amount of radiant energy actually used up in decomposition of the pentoxide. This is at least of the same order of magnitude as the 18 calories. More exact experimental data along this line will be of considerable interest.

A paper entitled "The Decomposition of Nitric Acid by Light," by Reynolds and Taylor, ' is of interest in connection with the present research. These investigators exposed sealed glass tubes of nitric acid out of doors for a year, and measured the extent of the decomposition by the pressure of oxygen liberated. They found that blue glass and colorless glass gave the same amount of decomposition, but that yellow glass prevented the photochemical action-an observation which is supported by the results given above. The photochemical decomposition occurred only in the gas phase, and was attributed to nitrogen pentoxide in equilibrium with the nitric acid. The authors were puzzled, however, by the fact that no decomposition resulted when concentrated sulfuric acid was added to the nitric acid, although the concentration of gaseous nitrogen pentoxide must have been increased. This unexpected result can be explained now because it has been shown that nitrogen pentoxide is decomposed by sunlight only if the dioxide is present, and the latter is removed from the gas phase by the sulfuric acid as fast as it is formed.

${ }^{1}$ Reynolds and Taylor, $J$. Chem. Soce, ror, 131 (1912). 
Another paper of interest in connection with the present research is that of Warburg and Leithauser ${ }^{1}$ on the infra-red spectra of the nitrogen oxides. The investigation extends from $2.4 \mu$ to $8.9 \mu$ and shows nitrogen pentoxide absorption bands at $2.82,2.91,3.39,3.89,4.29$ and $4.83 \mu$ with a particularly intense one at $5.81 \mu .30 \mathrm{~cm}$. of pentoxide at a pressure of $0.5 \mathrm{~mm}$. absorb $70 \%$ of the light of $5.81 \mu$ passing through it. Whether or not absorption at these lines results in decomposition of the gas will be the object of further experiments. It seems likely that such decomposition would result. Nitrogen dioxide has an absorption band at $3.43 \mu$ and an intense one at $6.12 \mu$, both of which are close to lines of nitrogen pentoxide.

The frequency of the theoretical absorption line calculated from the critical increment $1.16 \mu$ is exactly $;$ times as great as that of the intense absorption line $5.81 \mu$.

\section{Theoretical.}

The failure of nitrogen pentoxide to respond to radiant energy of wave length $1.16 \mu$, as calculated from the Lewis and Perrin theories, is of considerable interest. Several possible hypotheses might be advanced to explain this observation, a few of which are mentioned briefly. (See also the theoretical work of Tolman. $)^{2}$

1. There may be an absorption line at $1.16 \mu$ which is so narrow that a more powerful source of radiant energy or a still longer exposure may be required to give a detectable result. Further experiments along this line will be carried out and the spectrum in this region will be investigated.

2. The $1.16 \mu$ may represent a weighted average of some kind between infra-red and ultra-violet absorption.

$$
\begin{aligned}
& E=N h \nu \text { may have to be replaced by } \\
& E=N_{1} h \nu_{a}+N_{2} h \nu_{b}+N_{3} h \nu_{c} \\
& \text { where } N_{1}+N_{2}+N_{3}=N
\end{aligned}
$$

3. Einstein's law of photochemistry in which it is assumed that one quantum of energy $h \nu$ is required to disrupt one molecule may not be an exact law.

According to Millikan, ${ }^{3} h \nu$ represents the kinetic energy with which an electron is expelled (in the case of a metal) and the energy required to expel it is equal to $h \nu$ plus another quantity of energy which is required to loosen the electron.

$E$, the energy which must be absorbed as radiant energy in order to make a gram-molecule decompose, may be greater than $N h v$ in the same

\footnotetext{
1 Warburg and Leithauser, Ann. Physik., 28, 31:3 (190)!n.

Loc. cit.

s Millikan, Sience, 5x, 50.5 (1920).
} 
way. If so, the value of $\nu$ would become less and the significant wave length would be longer than the calculated $1.16 \mu$.

4. The assumption that activation is identical with decomposition in a monomolecular reaction may be invalid.

The following hypothesis is suggested as a possible explanation of the auto-catalytic effect of nitrogen dioxide. It absorbs blue light over a wide range and through fluorescence passes it out in the infra-red region where its absorption lines overlap those of nitrogen pentoxide and cause decomposition of the latter. Two assumptions, which have as yet no experimental support, are involved in this hypothesis; first, that nitrogen pentoxide is decomposed by the energy which it absorbs in the infra-red; and second, that the dioxide fluoresces, $i . e_{\text {., }}$ that light corresponding to its absorption lines in the infra-red is emitted when blue light is absorbed. The validity of these assumptions will be tested in the laboratory. This hypothesis demands that the dioxide should act as an auto-catalyst in the dark also, but the amount of energy between 400 and $460 \mu \mu$ emitted by a black body at temperatures between $0^{\circ}$ and $65^{\circ}$ is negligible compared with that in sunlight or lamplight. The increase in decomposition rate due to the presence of nitrogen dioxide in the experiments recorded in the preceding communication is, therefore, much less than the experimental error of observation.

If this hypothesis proves to be correct, it will lend valuable support to the radiant energy theory of catalysis. It will offer experimental facts in a simple gas-phase system to show that a substance can act as a catalyst by absorbing radiant energy, transforming it through fluorescence and giving it out at a particular frequency which is effective in bringing about a reaction. It suggests that other chemical reactions may be brought about by catalysts which absorb radiant energy over a wide range and emit it in a narrow region where it is effective. It suggests also that a catalyst of this type may be used together with radiation from an external source to bring about reactions which could not be brought about in a reasonable time by the catalyst alone or by the photochemical action alone.

The facts presented in this research give a concrete picture of the mechanism of at least one kind of "induction period." The decomposition of pure nitrogen pentoxide at $0^{\circ}$ is so slow as to be barely detectable, but after a while sufficient dioxide has been formed to give with light a fairly rapid reaction. It is easy to imagine other systems where one of the products acts as a transformer of radiant energy in such a way as to speed up the reaction after a considerable period of time has elapsed.

\section{Summary.}

1. The decomposition of nitrogen pentoxide at $0^{\circ}$ is a monomolecular, gas-phase reaction which offers unusual opportunities for studying the 
relation between thermal and photochemical reaction. The black-body radiation from the vessel walls at zero degrees causes a rate of reaction which is negligible in comparison with that produced by radiation received from a body, emitting white light and not in equilibrium with the system.

2. Calculations from the reaction velocity constants at different temperatures give 24,700 calories for the critical increment, $E$. From the expression $E=N h \nu, \lambda$ is found to be $1.16 \mu$. Light of this wave length, however, does not decompose nitrogen pentoxide within the limit of experimental observation.

3. Light in the region $400-460 \mu \mu$ accelerates the decomposition of nitrogen pentoxide.

4. Nitrogen pentoxide is not decomposed by light between 400 and $460 \mu \mu$ unless the dioxide is present.

5 . The auto-catalytic effect of nitrogen dioxide is negligible in the dark.

6. Hypotheses to explain these facts are offered and their relation to theories connecting chemical action with radiant energy are suggested.

Washington, D. C.

[Contribution hrom the Department of ImMunology, School of Hygtene ANd Public Health, Johns Hopkins University.]

\section{OBSERVATIONS ON THE ZINC ELECTRODE.}

By William C. MoOre.

Received October 6, 1920.

In a problem recently undertaken in this laboratory, it became necessary to measure small changes in concentration of zinc ion. As both the actual amount of zinc and the concentration changes involved were low (the former varying from the zinc contained in $10 \mathrm{cc}$. of $0.10 \mathrm{~N}$ zinc sulfate solution down to that contained in $10 \mathrm{cc}$. of $0.0025 \mathrm{~N}$ zinc sulfate solution, the latter varying by not more than a few per cent. of these amounts) it was thought that the zinc concentration cell might be used to accomplish the desired end.

A study of the literature, however, showed that all previous investigators had confined their attention either to dilute zinc amalgams of varying composition ${ }^{1}$ and constant concentration of electrolyte, or to zinc amalgams or amalgamated zinc, with zinc salt concentrations not less than $0.5 \mathrm{~N}^{2}$ Moreover, in practically all instances it was found necessary to take some sort of precaution to prevent the access of atmospheric oxygen to the electrolyte, and to the electrode. Thus, Kistiakowsky, ${ }^{3}$ who worked with normal zinc solutions, found that constant potentials

I Richards and Forbes, Carnegie Inst. Pub., 56 (1906).

${ }^{2}$ Mellenkamp, Phys. Rev., 29, 329-50 (1909).

' Kistiakowsky, Z. Elektrochem., I4, 113-121 (1908). 